\title{
USING ELECTRICAL CONDUCTANCE AS THE EVALUATION PARAMETER OF PAIN IN PATIENTS WITH LOW BACK PAIN TREATED BY ACUPUNCTURE- LIKE TENS
}

\author{
Ching-Sung Weng ${ }^{1.2}$, Yu-Show Tsal ${ }^{1}$, Che-Yen YAng ${ }^{3}$ \\ ${ }^{1}$ Department of Biomedical Engineering, Chung Yuan Christian University, Chung-Li \\ ${ }^{2}$ Chinese Medical Engineering Center, Chung Yuan Christian University, Chung-Li \\ ${ }^{3}$ Center for Traditional Chinese Medicine, Chang Gung Memorial Hospital, Tao-Yuang \\ Taiwan
}

\begin{abstract}
This study was to objectively evaluate the treatment effect of acupuncture-like transcutaneous electrical nerve stimulation (AL-TENS) on patients with low back pain. Fifty patients suffering from low back pain for at least three months were involved in the study from Chang Gung Memorial Hospital. The subjects were treated with TENS (100 Hz) on acupuncture points (BL23 and BL25). Two electrodes were placed on two right acupoints and two electrodes on the left. Pulse duration of electrical stimulation was fixed at $0.1 \mathrm{~ms}$. The intensity of stimulation was adjusted at a tolerable level for each subject. Patients were treated for twenty minutes per visit, three times a week for two weeks. Visual analog scale (VAS) was used to rank the degree of pain before and two weeks after the treatment. A device, the design of which is based on the Ryodoraku theorem, was used to measure the electrical conductance of 12 meridians and 2 acupoints (BL23 and BL25) on both sides of the subjects. The effect of age difference on the pain score was also evaluated with the electrical conductance. The correlation between the VAS and the electrical conductance in the patients before and after treatment of AL-TENS was determined by linear regression analysis. The results showed that: (1) after the AL-TENS treatment in this study, the electrical conductances of either meridians or acupuncture points increased with the decrease of VAS of the patients; (2) the correlation was higher in the acupoints than that in the meridians; (3) the correlation was higher in the younger group than that in the older group: (4) the effect of AL-TENS on the change of VAS is more related to the change of electrical conductances of acupoints than that of meridians. These findings suggest that electrical conductance of meridian or acupoint can be used to evaluate the degree of pain more objectively. especially in the younger patients.
\end{abstract}

Biomed Eng Appl Basis Comm, 2004 (August); 16: 205-212.

Keywords: Low back pain, Visual analog scale, Ryodoraku, Electrical conductance, Meridian, Acupuncture point

Received: March 18, 2004; Accepted: July 20, 2004 Correspondence: Ching-Sung Weng, Professor Department of Biomedical Engineering, Chung Yuan Christian University, 22 Pu-Jen, Pu-Chung Li, Chungli City, Taiwan

E-mail: csw@mail.be.cycu.edu.tw

\section{INTRODUCTION}

Pain is a common health problem in the world. Numerous treatments, such as transcutaneous electrical nerve stimulation (TENS), acupuncture-like transcutaneous electrical nerve stimulation (AL- 
TENS), percutaneous electrical nerve stimulation (PENS), acupuncture, electro-acupuncture (EA) are available for pain. TENS has most frequently been used for chronic pain [1-2]. The reason why TENS has a modulating effect on pain is that it is associated with blocked nociceptive transmission in the spinal cord [34]. The pain modulation of AL-TENS is associated with endorphin, which is released in response to afferent activity in the A delta fibers [5]. The modulating effect is usually activated by electrical stimulation with intensities as high as the patient can tolerate [6]. However, low and high intensities have been used in TENS and AL-TENS, respectively [7-8]. Another important parameter of electrical stimulation is "frequency". Low frequency $(2 \mathrm{~Hz}, 4 \mathrm{~Hz})$ and high frequency $(50,80,100,110 \mathrm{~Hz})$ have been used in TENS, AL-TENS, PENS, or EA for the study of pain [9-12]. It seems that there is no clear line to say which frequency is better and what intensity is good. Moreover, the site of stimulation is very critical for the outcome of treatment. Previous studies have shown that stimulation on acupuncture points is more effective than that on dermatomal and/or segmental areas $[5,13]$. It implies that AL-TENS could be better than TENS with the same condition of electrical parameters. Similarly, EA could be better than PENS. The evaluation of the treatment effect on pain is also an important issue. Usually the degree of pain can be evaluated by "visual analog scale" (VAS) as well as many other pain ranking methods [14-16]. In previous studies, increasing age has been associated with a higher frequency of incomplete or unscorable responses on the VAS [17-18]. Therefore VAS is a very subjective and qualitative method to evaluate pain. $A$ more objective and quantitative method is needed.

Earlier studies have shown that human health condition and diseases can be evaluated by the electrical conductance or impedance (inverse of conductance) of acupuncture points or meridians using the Ryodoraku technique [19-21]. Based on the Ryodoraku (meridian) theory, developed by Dr. Yoshio Nakatani, there are 12 meridians on the right and left sides of the human body. The property of ryodoraku can reflect the condition of certain organ(s) by analyzing and comparing their mutual relations and changes with micro electrical current [22]. The electrical state of the acupuncture points of the human subject is measured by a computerized testing instrument with a very low electrical current. From the traditional theory, Ryodoraku is a pathological phenomenon. The mechanism can be explained by the viscera-skin sympathetic nerve reflex. The impulses from the viscera radiate to the spinal cord. The reflex zones are then reflected onto the skin surface via the efferent sympathetic nerves and appear as a longitudinal connecting system, just like meridian lines.

It has been shown that meridians and acupuncture points have higher electrical conductance than other areas [20, 22-24]. Many studies have provided the mapping transforms between the electrical information given by Ryodoraku diagnosis and the patterns associated with various medical syndromes, signs, symptoms, and diseases. However, no research concerning the application of Ryodoraku theory in the diagnosis of pain has been reported. Based on the theory, the electrical conductances of meridians and acupoints as well as the VAS pain scores in the patients with low back pain were investigated in this paper.

\section{METHODS}

\subsection{Subjects}

This study was approved by the Chang Gung Memorial Hospital. Fifty patients with low back pain for at least three months were recruited in the study from the Hospital. All the subjects were in the ages between 20 and 69 years old. Subjects, who had a history of cardiovascular disease, liver disease, kidney disease, other organ diseases, and/or complaints of pain in the areas other than low back, were excluded. Patients, with problems in the care of the electrical stimulation and with skin allergic to electrodes, were also not involved in the study.

\subsection{TENS Procedure}

Fifty subjects were treated with TENS on acupuncture points (BL23 and BL25). The TENS device used in this study is just like other TENS. Two rubber electrodes ( $2 \mathrm{~cm}$ in diameter) were placed on two right acupoints and two electrodes on the left. Frequency was fixed at $100 \mathrm{~Hz}$. Pulse duration of electrical stimulation was fixed at $0.1 \mathrm{~ms}$. The intensity of stimulation was adjusted at a tolerable level for each subject. Patients were treated for twenty minutes per visit, three times a week for two weeks. Each treatment was performed by the same physician.

\subsection{Measurements}

The degree of pain of all the subjects was evaluated with visual analog scale ( 0 -no pain, 10 -the worst pain the patient had ever experienced) by the same doctor before and after treatment. The electrical conductances of 24 acupuncture points (Ryodoraku points) in the twelve left meridians and the twelve right meridians were measured with a device (C.Y. Biotech, with Taiwan's Patent), the design of which is based on the Ryodoraku theory. This device has been used in previous studies [25-26]. Also, the electrical 
conductances of 4 acupuncture points (BL23 and BL25) in the area of low back pain were measured. Before measuring, the device was well calibrated to assure its reproducibility. Each time of measurement was taken by the same research assistant to avoid human artifact error. The room temperature was kept between 23 and $26^{\circ} \mathrm{C}$; and the humidity was kept constant by air conditioning. Readings of electrical conductance were recorded from both groups before and two weeks after treatment procedure. The conductance values were calculated with voltage supplied by the device and currents measured in meridians or acupuncture points and expressed as the scale between 0 (zero conductivity) and 100 (maximum conductivity). The mean conductance value of 24 meridians in each subject was calculated. Also, the mean conductance value of 4 acupoints (BL23 and BL25) in each subject was calculated. The average and standard error of such values $(n=50)$ were then determined and expressed as "Mean $\pm \mathrm{SE}$ " for later statistical analysis.

\subsection{Statistical Analysis}

The pain scores of VAS before and after the treatment were compared with non-parametric Wilcoxon's signed rank test. The difference between the conductance values (Mean $\pm S E$ ) of the patients measured before and after the AL-TENS procedure was analyzed by paired $t$ test. The difference between the electrical conductances of meridians and that of acupuncture points either before or after the treatment was also analyzed by paired t test. A statistical significance was recognized as p-value $<0.05$. Regression analysis was used to evaluate the correlation between VAS and electrical conductance. As mentioned earlier, the VAS for the assessment of changes in pain is not sufficiently sensitive to detect age differences [14]. The data were divided into two different age groups, that the all subjects were split at the median age of 41 years, for linear regression and correlation analysis.

\section{RESULTS}

Fifty subjects were aged between 20 and 69 years with a mean age of $48.7 \pm 14.3$ years. As shown in Table I, there was a significant difference of VAS before and after treatment $(p<0.01)$. As shown in Table II, there was a significant difference of electrical conductance of the 24 meridians before and after treatment $(p<0.05)$; also, there was a significant difference of electrical conductance of four acupoints before and after treatment $(p<0.01)$. The electrical conductances of meridians and that of acupuncture
Table I. Visual analog scale (VAS) pain scores (Mean $\pm S D$ ) in patients before and after AL-TENS treatment

\begin{tabular}{ll}
\hline & VAS \\
\hline before & $5.13 \pm 1.54^{* *}$ \\
after & $2.42 \pm 1.06^{* *}$ \\
\hline$* * p<0.01$ &
\end{tabular}

Table II. Electrical conductances (Mean $\pm \mathrm{SE}$ ) of meridians and acupoints in patients before and after AL-TENS treatment

\begin{tabular}{lll}
\hline & 24 Meridians & 4 Acupoints \\
\hline before & $44.79 \pm 12.52 \mathrm{a}, \mathrm{c}$ & $34.88 \pm 12.34 \mathrm{~b}, \mathrm{c}$ \\
after & $66.38 \pm 10.91 \mathrm{a}, \mathrm{d}$ & $56.50 \pm 10.39 \mathrm{~b}, \mathrm{~d}$ \\
\hline
\end{tabular}

* a, c, d: $p<0.05 ; * * b: p<0.01$

points either before or after the treatment were significantly different $(p<0.05)$. As shown in Figure 1, the VAS and the electrical conductance (EC) of the 24 meridians were correlated in all the fifty patients either before treatment $\left(\mathrm{VAS}=7.080-0.070^{*} \mathrm{EC} ; \mathrm{r}=-0.584\right.$, $\mathrm{p}<0.001$ ) or after treatment (VAS $=5.197-0.059 * \mathrm{EC}$; $r=-0.690, p<0.001)$. As shown in Figure 2, the VAS and the electrical conductance of four acupoints were correlated in all the fifty patients either before treatment $\left(\mathrm{VAS}=7.841-0.090^{*} \mathrm{EC} ; \mathrm{r}=-0.733, \mathrm{p}<0.001\right)$ or after treatment (VAS $=5.128-0.053 * \mathrm{EC} ; \mathrm{r}=-0.722$, $\mathrm{p}<0.001$ ). As shown in Figure 3, the VAS and the electrical conductance (EC) of the 24 meridians were correlated in the older group either before treatment $(\mathrm{VAS}=5.598-0.042 * \mathrm{EC} ; \mathrm{r}=-0.459, \mathrm{p}<0.05)$ or after treatment (VAS=4.937-0.055*EC; $r=-0.668, p<0.05$ ). As shown in Figure 4, the VAS and the electrical conductance of four acupoints were correlated in the older group either before treatment (VAS $=5.798$ $0.044 * E C ; r=-0.462, p<0.05)$ or after treatment (VAS $=4.982-0.055^{*} \mathrm{EC} ; \mathrm{r}=-0.523, \mathrm{p}<0.01$ ). As shown in Figure 5, the VAS and the electrical conductance (EC) of the 24 meridians were correlated in the younger group either before treatment (VAS $=8.978$ $\left.0.106^{*} \mathrm{EC} ; \mathrm{r}=-0.823, \mathrm{p}<0.01\right)$ or after treatment $\left(\mathrm{VAS}=6.101-0.076^{*} \mathrm{EC} ; \mathrm{r}=-0.712, \mathrm{p}<0.01\right)$. As shown in Figure 6, the VAS and the electrical conductance of four acupoints were correlated in the younger group either before treatment (VAS=9.077-0.113*EC; $r=-$ $0.911, p<0.001)$ or after treatment (VAS $=7.148$ $\left.0.085^{*} \mathrm{EC} ; \mathrm{r}=-0.858, \mathrm{p}<0.001\right)$. 


\section{DISCUSSION}

The aim of this research was to determine the correlation between the electrical conductances of meridians or acupuncture points and VAS pain scores in the patients with low back pain. Also, the effect of acupuncture-like TENS on the patients was evaluated with electrical conductance and VAS. From the results, VAS pain score indicated that the AL-TENS was effective. Stimulation of TENS on acupuncture points changes the electrical conductance of meridians and acupuncture points, and regulates the central nervous system (CNS). The regulation of the human health condition can be likened to the regulation of homeostatically controlled variables, such as bioelectricity or bio-energy. Being homeostatic, mammals are able to maintain relatively constant internal parameters in the face of variable and often extreme ambient changes. This fact is easily accomplished by means of coupled afferent and efferent mechanisms. Sensory receptors in the skin, muscle, nerve, organ, and elsewhere continuously send messages (such as electrical impulses) to the CNS. The $\mathrm{CNS}$ in turn combines this information with other relevant information (activity level, needs of various tissues, cognitive information) and adjusts the gain of some subset of possible effect or mechanism (pain in local area, blood flow in superficial veins, breathing rate, perspiration, etc). The result is that the abnormal condition such as body pain can be accomplished via adjustments in both body fluid (including blood) regulation and autonomic responses. To our knowledge, the role of the brain (especially the hypothalamus) in the regulation of energy or health condition is well established in animals. In humans, substantial molecular and neuropharmacologic evidence attest to the importance of the central nervous system in maintaining an energy balance. The basic promise of energy medicine is that energetic processes, including electrical and magnectic processes, vibrational resonance, and bio-photon emission, are essential to the life process. Bio-energy functions are carrier of "bio-information" and are crucial to biological self-regulation [27]. Bio-energy has been studied, by measuring the electrical conductances of meridians and/or acupuncture points, in healthy and diseased people $[23,28]$. Therefore, the central nervous system plays an important role in the regulation of bio-energy as well as the meridian system. The pathogenesis of pain has been suggested to be intimately linked to the regulation of the sympathetic nervous system. Excitation of a sympathetic response may alter the afferent input to the CNS and evoke analgesic effect [29]. TENS can usually affect peripheral conduction, excite the

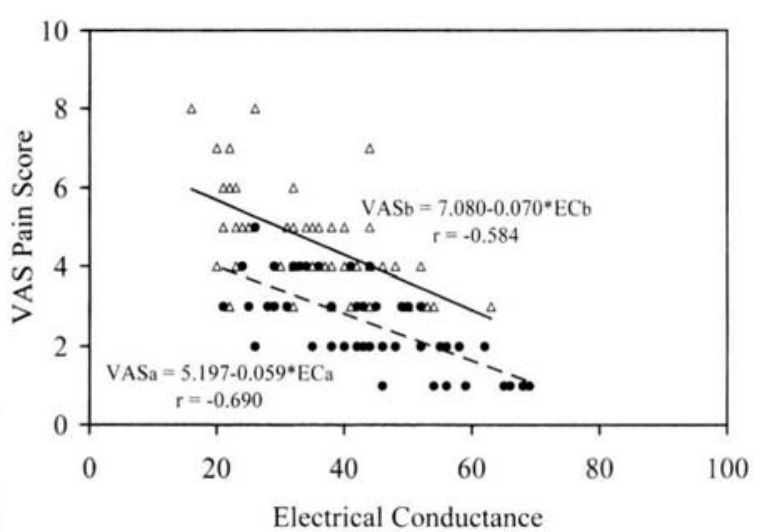

Fig 1. Linear regression analysis for all the patients $(n=50)$ treated with AL-TENS and measured of electrical conductances on meridians. There are $\mathbf{5 0}$ data points for all the patients before (in triangle) and after (in solid circle) treatment; 100 data points in total. For each data point, the subject's VAS is on the $Y$-axis and his average of electrical conductances of 24 meridians is on the $X$-axis. The solid line and broken line are the least-squares lines for the group before and after treatment, respectively.

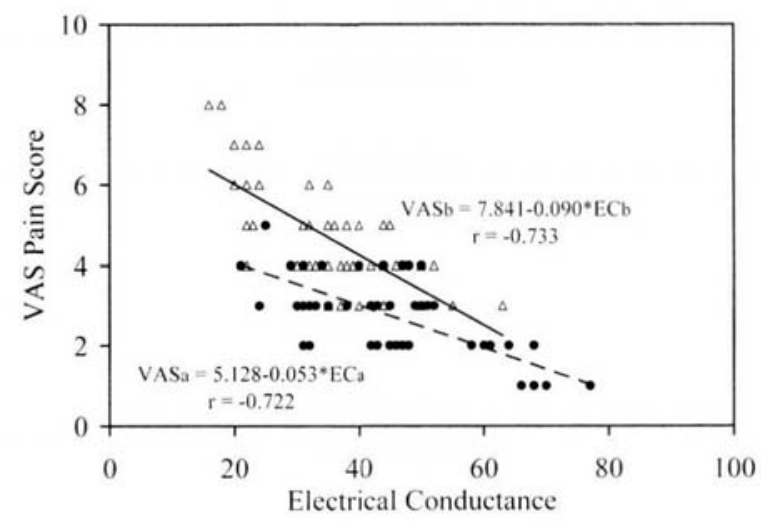

Fig 2. Linear regression analysis for all the patients $(n=50)$ treated with AL-TENS and measured of electrical conductances on acupoints. There are 50 data points for all the patients before (in triangle) and after (in solid circle) treatment; 100 data points in total. For each data point, the subject's VAS is on the $Y$-axis and his average of electrical conductances of 4 acupoints is on the $X$-axis. The solid line and broken line are the least-squares lines for the group before and after treatment, respectively. 


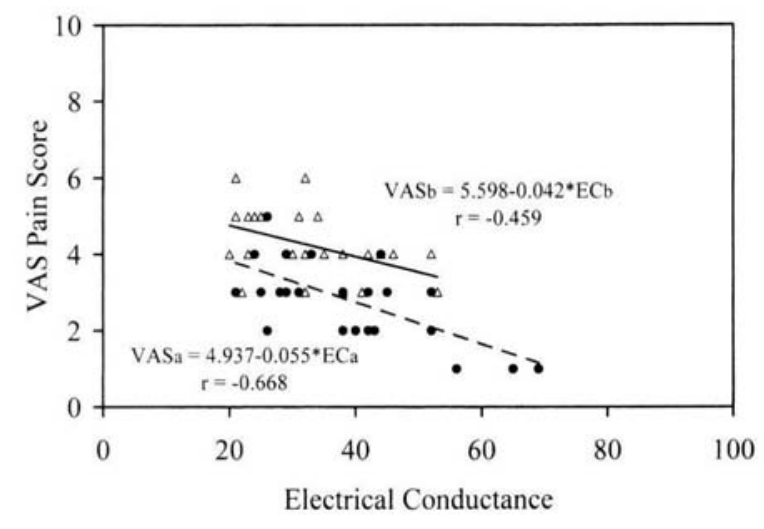

Fig 3. Linear regression analysis for the older group patients $(n=25)$ treated with AL-TENS and measured of electrical conductances on meridians. There are $\mathbf{2 5}$ data points for the group before (in triangle) and after (in solid circle) treatment; 50 data points in total. For each data point, the subject's VAS is on the Y-axis and his average of electrical conductances of 24 meridians is on the $X$ axis. The solid line and broken line are the leastsquares lines for the group before and after treatment, respectively.

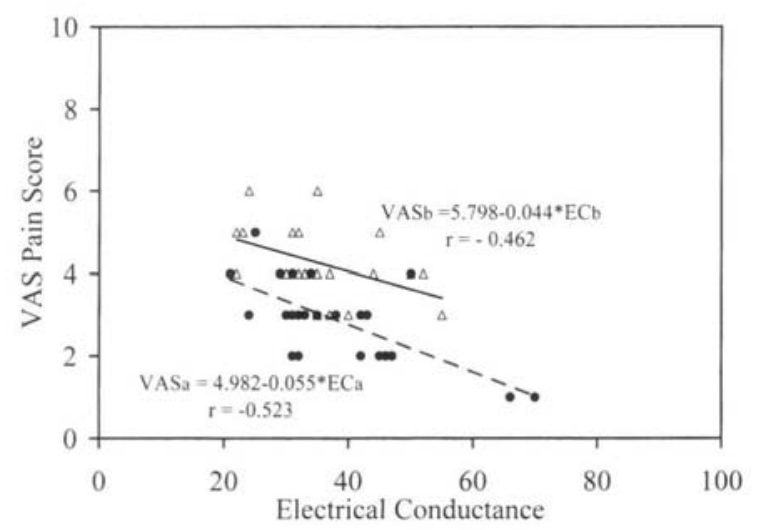

Fig 4. Linear regression analysis for the older group patients $(n=25)$ treated with AL-TENS and measured of electrical conductances on acupoints. There are $\mathbf{2 5}$ data points for the group before (in triangle) and after (in solid circle) treatment; 50 data points in total. For each data point, the subject's VAS is on the $Y$-axis and his average of electrical conductances of 4 acupoints is on the $X$ axis. The solid line and broken line are the leastsquares lines for the group before and after treatment, respectively. sympathetic nerve, and mediate hypoalgesia [30], while AL-TENS has an even stronger effect on the conductance of meridian system. This is besides its effect on the CNS.

Many earlier studies have shown that the nervous system is somewhat related to the meridian system. Usually, needles, heat, or electrical stimulation on acupuncture points can give rise to nervous impulses and regulate the body or organ functions. A good body function often has a nice conductivity of heat or electrical energy. Our study demonstrated that the measurement of meridian electrical conductance could be a way to evaluate how the meridian (even the nervous system) is regulated before and after the $\mathrm{AL}$ TENS treatment. As the results showed, electrical conductance of meridian or acupuncture point increased when the level of pain was claimed to decrease in patients with low back pain.

Patients with chronic low back pain frequently suffer from tiredness and lack of strength as the subjects in the study. Tiredness or lack of strength is one of the symptoms of qi vacuity syndrome which is correlated to the decrease of skin conductance [31]. Although the device they used is based on Dr. Voll's research [32], the skin conductance they measured is still related to the meridian conductance measured on the skin surface of the acupuncture point in our study. In that case, skin electrical resistance, skin moisturization, and sub-skin tissue water content can be important when taking the measurement. The water content of stratum corneum, which in turn is influenced by sweat gland function, influences skin resistance. The sweat gland is a target organ of the peripheral autonomic system. By measuring the electrical conductance of the skin over acupuncture point, the function of the peripheral autonomic system could be measured on a digital scale [33]. Sweat glands are innervated by cholinergic fibers. Increased intradermal acetylcholine causes the secretion of sweat glands, which in turn leads to an increase of skin conductance and decrease of skin electrical resistance. By measuring (regional) skin resistance, the function of peripheral (regional) autonomic innervation can be evaluated indirectly [34-35]. Moreover, the distribution of the intradermal cholinergic fibers is not always the same. This provides the reason why the electrical conductances measured in this research were different (i.e., the standard deviation of mean of meridian electrical conductance) in the twelve meridians of the same subject. The patient with low back pain usually has a weak sympathetic nervous pulse in some meridian regions and also showed the worse conductance. This is the major reason why the conductance was lower in the low back pain area, with 4 acupoints (BL23, BL25) on, than that in the 24 meridians. 


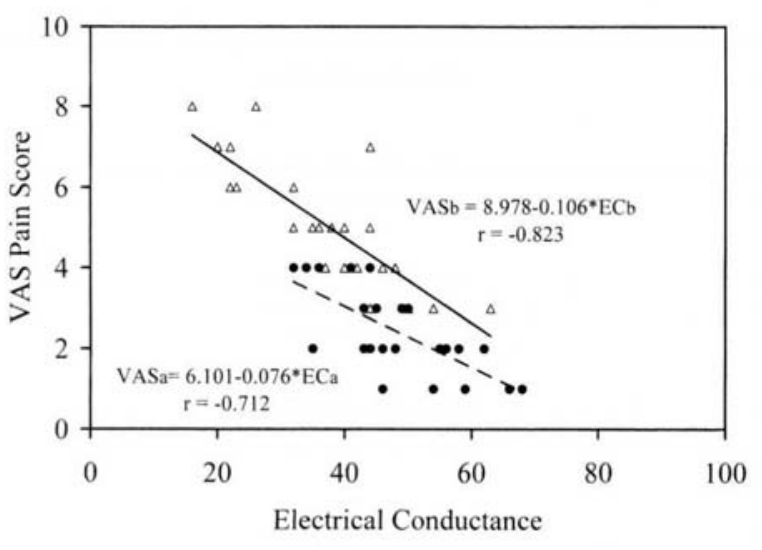

Fig 5. Linear regression analysis for the younger group patients $(n=25)$ treated with AL-TENS and measured of electrical conductances on meridians. There are 25 data points for the group before (in triangle) and after (in solid circle) treatment; 50 data points in total. For each data point, the subject's VAS is on the Y-axis and his average of electrical conductances of 24 meridians is on the $X$ axis. The solid line and broken line are the leastsquares lines for the group before and after treatment, respectively.

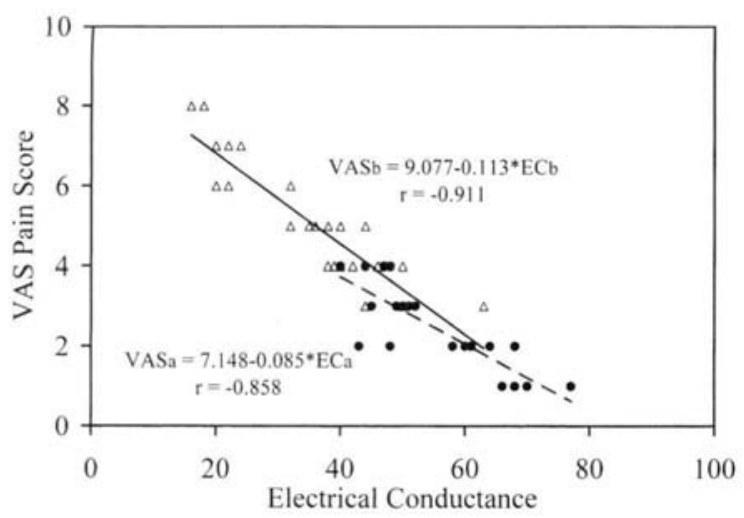

Fig 6. Linear regression analysis for the younger group patients $(n=25)$ treated with AL-TENS and measured of electrical conductances on acupoints. There are 25 data points for the group before (in triangle) and after (in solid circle) treatment; 50 data points in total. For each data point, the subject's VAS is on the $Y$-axis and his average of electrical conductances of 4 acupoints is on the $X$ axis. The solid line and broken line are the leastsquares lines for the group before and after treatment, respectively.
Furthermore, the electrical conductance (inverse of impedance) can be influenced by acupuncture or other stimulations [36]. After the AL-TENS treatment in this study, the electrical conductances of either meridians or acupuncture points increased with the decrease of VAS of the patients. The relationship between the electrical conductance and VAS pain score could be simply evaluated by linear regression analysis. The correlation was higher in the acupoints than that in the meridians. Also, the correlation was higher in the younger group than that in the older group. This demonstrated that age difference is an issue when pain is evaluated by VAS pain score [37]. The effect of AL-TENS on the percentage change of VAS is more related to the percentage change of electrical conductances of acupoints than that of meridians.

In summary, the findings in this paper suggest that the electrical conductance of acupoints in the area of pain can be used to quantitatively evaluate the degree of pain other than using the qualitative VAS pain score either before or after AL-TENS treated patients with low back pain.

\section{ACKNOWLEDGEMENTS}

The authors thank Professor Wei-Chi Hu for his technical support; Lecturer David Cedric Bennette for his valuable comments to the final version of the manuscript.

\section{REFERENCES}

1. Gersh MR and Wolf SL: Application of transcutaneous electrical nerve stimulation in the management of patients with pain: state of-the-art update. Phys Ther 1985; 65:314-322.

2. Mannheimer JS and Lampe GN: Clinical transcutaneous electrical nerve stimulation. Philadelphia: FA Davis, 1984.

3. Garrison DW and Foreman RD: Effects of transcutaneous electrical nerve stimulation (TENS) on spontaneous and noxiously evoked dorsal horn cell activity in cats with transected spinal cords. Neurosci Lett 1996; 216:125-128.

4. R and Wall PD: Pain mechanisms: a new theory. Science 1965; 150:971-979.

5. Chen L, Tang J, White PF, Sloninsky A, Wender RH, Naruse R and Kariger R: The effect of location of transcutaneous electrical nerve stimulation on post-operative opioid analgesic requirement: acupoint versus nonacupoint stimulation. Anesth Analg 1998; 87:1129-1134. 
6. Bushnell MC, Marchand S, Tremblay $\mathrm{N}$ and Duncan $\mathrm{GH}$ : Electrical stimulation of peripheral and central pathways for the relief of musculoskeletal pain. Can J Physiol Pharmacol 1991; 69:697-703.

7. Walsh DM: TENS clinical applications and related theory. New York, NY:Churchill Livingstone, 1997.

8. Walsh DM and Baxter GD: Transcutaneous electrical nerve stimulation (TENS): a review of experimental studies. Eur J Phys Med Rehabil 1996; 6:41-51.

9. Ghoname EA, White PF, Ahmed HE, Hamza MA, Craig WF and Noe CE: Percutaneous electrical nerve stimulation: an alternative to TENS in the management of sciatica. Pain 1999; 83:193-199.

10. Grant DJ, Bishop-Miller J, Winchester DM, Anderson $M$ and Faulkner S: A randomized comparative trial of acupuncture versus transcutaneous electrical nerve stimulation for chronic back pain in the elderly. Pain 1999; 82:913.

11. Lin JG, Lo MW, Wen YR, Hsieh CL, Tsai SK and Sun WZ: The effect of high and low frequency electroacupuncture in pain after lower abdominal surgery. Pain 2002; 99:509-514.

12. Sluka KA, Bailey K, Bogush J, Olson R and Ricketts A: Treatment with either high or low frequency TENS reduces the secondary hyperalgesia observed after injection of kaolin and carrageenan into the knee joint. Pain 1998; 77:97102.

13. Takeshige $C$, Sato $T$, Mera $T$, Hisamitsu $T$ and Fang J: Descending pain inhibitory system involved in acupuncture analgesia. Brain Res Bull 1992; 29:617-634.

14. Gagliese L and Katz J: Age differences in postoperative pain are scale dependent: a comparison of measures of pain intensity and quality in younger and older surgical patients. Pain $2003 ; 103: 11-20$.

15. Huskisson EC: Visual analogue scales. In: Melzack $\mathrm{R}$, Ed. Pain measurement and assessment. Raven Press, New York, USA, 1983; 33-37.

16. Jensen MP and Karoly P: Self-report scales and procedures for assessing pain in adults. In: Turk DC, Melzack R, Eds. Handbook of pain assessment. Guilford Press, New York, USA, 2001; 135-151.

17. Gagliese L and Melzack R: Age differences in the quality of chronic pain: a preliminary study. Pain Res Manage 1997; 2:157-162.

18. Herr KA and Mobily PR: Comparison of selected pain assessment tools for use with the elderly. Appl Nurs Res 1993; 6:39-46.

19. Becker RQ, Reiochmanis $M$ and Marino A: Electrophysiological correlates of acupuncture points and meridians. Psychoenergetic Systems
1976; 1(105):195-212.

20. Chen KG: Electrical properties of meridians: with an overview of the electrodermal screening test. IEEE Eng in Med and Biol 1996; 3:58-63.

21. Sullivan SG, Eggleston DW, Martinoff JT and Kroening RJ: Evoked electrical conductivity on the lung acupuncture points in the healthy individuals and confirmed lung cancer patients. Am J Acupunct 1985; 13:63-68.

22. Reichmanis M, Andrew A and Becker RQ: DC skin conductance variation at acupuncture loci. Am J Chin Med 1976; 4:69-72.

23. Lam F, Jr, Tsuei JJ and Zhao Z: Studies on the bioenergetic measurement of acupuncture points for determination of correct dosage of allopathic or homeopathic medicine in the treatment of diabetes mellitus. Am J Acupunct 1990; 18:127-133.

24. Zhu ZX: Research advances in the electrical specificity of meridians and acupuncture points. Am J Acupunct 1981; 9:203-216.

25. Wang $\mathrm{CN}$, Weng $\mathrm{CS}, \mathrm{Hu} \mathrm{WC}$, Chang $\mathrm{YH}$ and Lin JG: The development of new ryodoraku neurometric patterns. J Med Bio Eng 2002; 22(S):99-106 (In Chinese).

26. Weng CS, Wang CN, Hu WC and Sun MF: Evaluation of acupuncture treatment of low back pain using a new ryodoraku neurometric system. J Chin Med Sci 2003; 3(1):65-72 (In Chinese).

27. Tsuei JJ: The science of acupuncture: theory and practice. IEEE Eng in Med and Biol 1996; 3:52-57.

28. Tusei JJ, Chung C, Lam F and Jr. Mi M: Studies of bioenergy in healthy subjects. Am J Acupunct $1988 ; 16: 125-134$.

29. Willis WD and Westlund KN: Neuroanatomy of the pain system and of the pathways that modulate pain. J Clin Neurophysiol 1997; 14:2-31.

30. Walsh DM, Lowe AS, McCormack K, Willer JC, Baxter GD and Allen JM: Transcutaneous electrical nerve stimulation: effect on peripheral nerve conduction, mechanical pain threshold, and tactile threshold in humans. Arch Phys ed Rehabil 1998; 79:1051-1058.

31. Yu HM, Chang HH, Liou SH, Li SF, Hou MM and Chen MF: The correlation between skin electrical conductance and the score of qi vacuity. Am J Chin Med 1998; 26:283-290.

32. Voll R: The phenomenon of medicine testing in electroacupuncture according to Voll. Am J Acupunct 1980; 8:97-104.

33. Yamamoto Y: Measurement and analysis of skin electrical impedance. Acta Derm Venerol(stockh) 1994; 185:34-38.

34. Ryder RE, Kennedy RL and Newrick PG: Autonomic denervation may be a prerequistite diabetic neuropathic foot ulceration. Diabet Med 1990; 7:726-730. 
35. Tagami, H: Quantitative measurements of water concentration of the stratum corneum in vivo by high frequency current. Acta Derm Venerol(stockh) supp 1994; 185:29-33.

36. Zhang W, Xu R and Zhu Z: The influence of acupuncture on the impedance measured by four electrodes on meridias. Acupuncture \& ElectroTherapeutics Res, Int J 1999; 24:181-188.

37. Benesh LR, Szigeti E, Ferraro FR and Gullicks JN: Tools for assessing chronic pain in rural elderly women. Home Healthcare Nurse 1997; 15:207-211. 